\title{
Research on the Control System of Intelligent Electric Assisted Knee Joint
}

\author{
Li Feng ${ }^{1}$, Wang Tianrun ${ }^{2}$, Zhang Dedi ${ }^{2}$, Liu Ning ${ }^{2}$ \\ ${ }^{1}$ Beijing Jingbo Modern Prosthetic Orthosis Technology Co., Ltd., Beijing, China \\ ${ }^{2}$ Beijing Key Laboratory of High Dynamic Navigation Technology, Beijing Information Science and Technology University, Beijing, China
}

\section{Email address:}

borneol@126.com (Li Feng),wqwqwq604@hotmail.com (Wang Tianrun),zdd15600991921@gmail.com (Zhang Dedi), ning.liu@bistu.edu.cn (Liu Ning)

\section{To cite this article:}

Li Feng, Wang Tianrun, Zhang Dedi, Liu Ning. Research on the Control System of Intelligent Electric Assisted Knee Joint. Science Discovery. Vol. 7, No. 6, 2019, pp. 419-428. doi: 10.11648/j.sd.20190706.18

Received: November 3, 2019; Accepted: December 3, 2019; Published: December 9, 2019

\begin{abstract}
Aiming at the problem that the traditional electric assisted knee joint is high cost, non-intelligent, cannot automatically adjust the assist strength, a new electric assisted knee joint control system was developed in this paper, which is divided into three parts: Android control terminal, prosthetic control module and prosthetic mechanical structure. The Android control terminal performs data interaction with the prosthetic control module via Bluetooth, controls the movement of the mechanical part. The system comprises a power module, a sensor module, a data processing module, a control module, an information interaction module and a control terminal module, after the testing, all parts of the system work normally, and has the functions of real-time automatic adjustment of the knee joint assisting strength, real-time remote manual control assisting strength, real-time data uploading and other functions.
\end{abstract}

Keywords: Electric Assisted Knee Joint, Real-time Power Adjustment, Remote Control

\section{智能电动助力膝关节控制系统研究}

李锋 ${ }^{1}$, 王天润 ${ }^{2}$, 张德帝 ${ }^{2}$, 刘宁 ${ }^{*}$

${ }^{1}$ 北京精博现代假肢矫形器技术有限公司, 北京, 中国

2高动态导航技术北京市重点实验室, 北京信息科技大学, 北京, 中国

邮箱

borneol@126.com (李锋), wqwqwq604@hotmail.com(王天润), zdd15600991921@gmail.com（张德帝）, ning.liu@bistu.edu.cn（刘宁）

摘要: 针对传统电动助力膝关节成本高、非智能、无法自动调节助力强度的问题, 本文研制了一种新的电动助力膝关 节控制系统，系统分为Android控制终端、假肢控制模块、假肢机械结构三部分。Android控制终端通过蓝牙与假肢控 制模块进行数据交互, 控制机械部分运动, 进行助力行走。系统包含电源模块、传感器模块、数据处理模块、控制模 块、信息交互模块、控制终端模块，经试验此系统各部分工作正常，且具有实时自动调节膝关节助力大小、实时远程 手动控制助力大小、数据实时上传等功能。

关键词: 助力膝关节, 实时助力调节, 远程控制 


\section{1. 引言}

由于战争, 工伤, 交通事故和自然灾害等频繁发生, 膝盖截肢者的数量每年增加数万人。根据第二次全国残疾 人抽样调查的数据显示, 全国各类残疾人的总数为 8296 万人, 占全国人口总数的 $6.34 \%$, 其中肢体残疾人数为 2412 万人, 占 $29.07 \%[1]$, 是残疾人中最大的群体。而中国康 复医学会2006年统计的大腿截肢患者达到 1725 万, 约占肢 体残疾总人数的 $71.5 \%[2]$ 。随着经济的发展, 我国人民生 活水平的提高, 残疾人对于生活质量的要求也越来越迫切。 而在当前的医疗水平下, 尚不能达到残肢再生的地步, 因 此安装假肢就成为了截肢患者恢复行走能力[3], 进行各种 日常活动, 重返社会正常工作生活的唯一方法。

膝关节是一种高度非线性、时变、强耦合的系统[4]。 作为下肢假肢最重要的部分 [5], 膝关节假肢要能够根据步 态变化自动调节控制参数, 保证关节在支撑期有较好的稳 定性, 在摆动期有较好的灵活性[6]。针对传统电动助力膝 关节非智能、无法自动调节助力强度、成本高的问题, 研 制了一种新的电动助力膝关节控制系统。系统包含电源模 块、传感器模块、数据处理模块、控制模块、信息交互模 块、控制终端模块, 具有实时自动调节膝关节助力大小、 实时远程手动控制助力大小、数据实时上传等功能。

\section{2. 系统架构与工作原理}

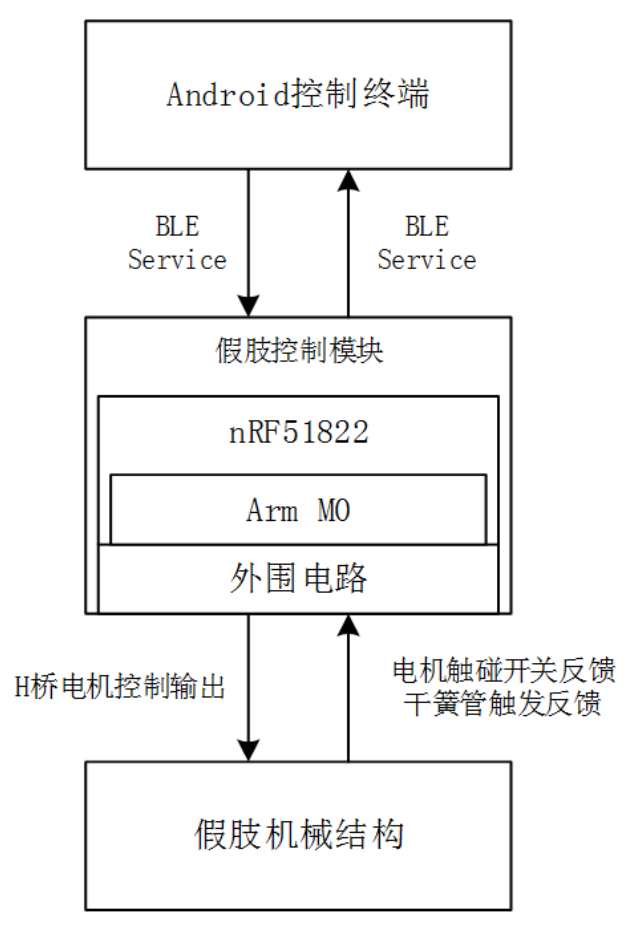

图1 系统整体架构图。

本文研制了一种基于BLE的智能电动助力膝关节, 具 有自动、远程手动调节助力档位, 档位参数可调的功能。 响应迅速, 穿戴舒适。
如图1所示，整个系统分为Android控制终端、假肢控 制模块、假肢机械结构三部分。Android控制终端模块是 通过QT $(\mathrm{C}++)$ 在手机终端上开发的App, 通过BLE Service 的方式与假肢控制模块进行数据交互。用户可以通过手机 直接控制假肢上的助力电机, 进而控制助力大小, 可以任 意修改每一种运动状态对应的助力档位, 也可以直接在手 机上获取当前的助力大小、运动状态。

假肢控制模块是在nRF51822(C)上开发的基于BLE协 议栈的系统[7]。系统通过BLE与Android端进行信息交互, 实时上传运动数据与助力数据, 实时响应Android端发出 的控制指令。系统通过两路 $\mathrm{H}$ 桥输出控制假肢上的电机正 反转及转动时间, 通过接收假肢上的电机触碰开关和运动 中磁铁触发的干簧管的反馈来采集运动信息, 进而执行相 应控制策略, 实时根据运动状态以及预设指令调整膝关节 的助力大小。

\section{Android端通讯原理与界面设计}

Android端APP开发基于BLE协议，在QT IDE下采用 $\mathrm{C}++$ 语言设计完成, 生成APK文件, 运行平台为Android 9.0。 APP的架构、各模块之间的通信、APP与假肢控制模块的 通信如图2所示。

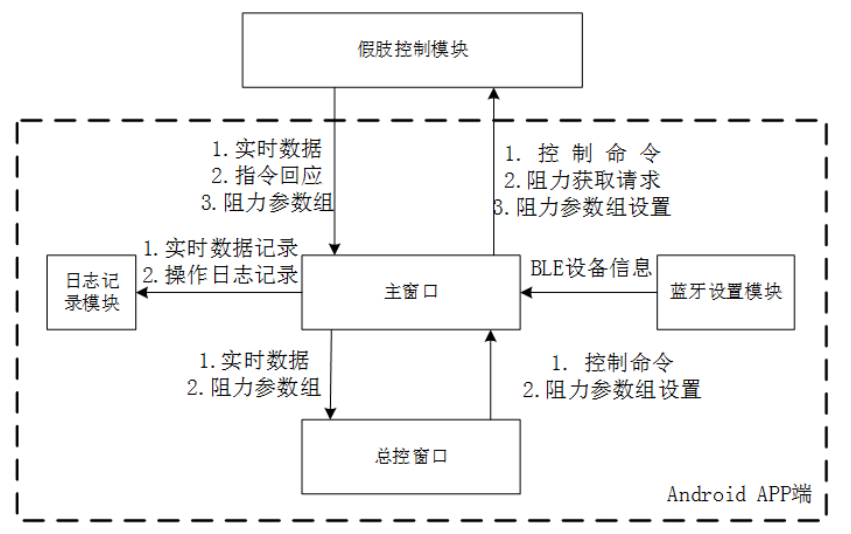

图2 APP的架构、各模块之间的通信、APP与假肢控制模块的通信Android 端与假肢控制端的连接与通信。

Android端与假肢控制端的通信采用基于BLE协议的 方式, 通过BLE的service进行数据的交互。连接流程如图 3 所示。

主窗口在接收到蓝牙设置模块发送的BLE从机设备 信息后即可开始连接设备, 连接成功后, 会搜索设备提供 的Service及其UUID, 经过一一匹配后找到所需的串口通 讯服务, 然后进一步搜索服务中所包含的关键字 TX、RX, 以及关键字的Descriptor[8]。 $\mathrm{RX}$ 为从机的输入接口, 通过 writeCharacteristic $($ 函数写入相关通讯信息, $\mathrm{TX}$ 为从机的 输出接口, 含有一个Descriptor, 需要向其中写入正确的关 键字才可启用其通知功能, 然后在接收到TX特征值发生 改变的信号后读取特征值内容即可[9]。

上位机与下位机之间所有的信息交互都以主窗口为 接口。主窗口接收下位机上传的实时运动与控制数据、指 
令回应、阻力参数组并进行相应的处理与显示。交互数据 的结构为: \{数据类型, 数据内容 $\}$, 具体内容如表 1 所示。

表1 Android端与假肢控制端的通讯交互数据格式。

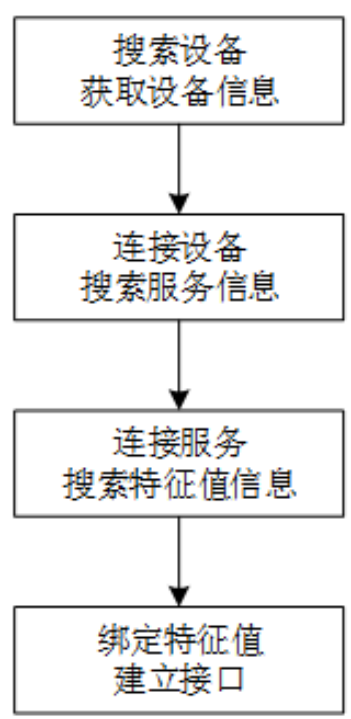

图3 BLE设备连接的流程。

\begin{tabular}{|c|c|c|c|c|}
\hline 传递方向 & 数据内容 & $\begin{array}{l}\text { 长 } \\
\text { 度 }\end{array}$ & 头 & 帧结构 \\
\hline \multirow{3}{*}{ 上行 } & $\begin{array}{l}\text { 下位机 实 } \\
\text { 时数据 }\end{array}$ & 5 & & \multirow{5}{*}{ 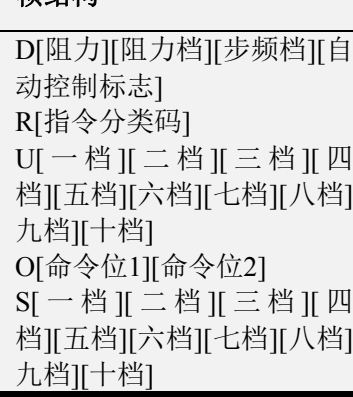 } \\
\hline & 指令回应 & 2 & $0 \times 52$ & \\
\hline & $\begin{array}{l}\text { 当 前 的 阻 } \\
\text { 力参数组 }\end{array}$ & 11 & $0 \times 55$ & \\
\hline \multirow[b]{2}{*}{ 下行 } & 控制命令 & 3 & $0 \times 4 F$ & \\
\hline & $\begin{array}{l}\text { 阻力参 数 } \\
\text { 组设置 }\end{array}$ & 11 & $0 \times 53$ & \\
\hline
\end{tabular}

\subsection{Android APP内各模块功能}

\subsection{1. 日志记录模块}

日志记录模块负责接收主窗口传来的记录指令, 进行 相应的日志记录。日志分为两种: 操作日志和数据记录, 通过不同的函数进行相应的记录。

日志的格式见表 2 , 记录的内容见表3。

表2 日志的格式。

\begin{tabular}{|c|c|c|}
\hline 日志 & 项目 & 格式 \\
\hline $\begin{array}{l}\text { 操作日志 } \\
\text { 数据日志 }\end{array}$ & $\begin{array}{l}\text { 文件名称 } \\
\text { 内容 } \\
\text { 文件名称 } \\
\text { 内容 } \\
\end{array}$ & $\begin{array}{l}\text { log + MM-dd.txt } \\
\text { dd-hh:mm:ss + 操作内容 }+ \text { 自动换行 } \\
\text { data + MM-dd.txt } \\
\text { dd-hh:mm:ss + 数据+ 自动换行 } \\
\end{array}$ \\
\hline \multicolumn{3}{|c|}{ 表3 日志记录内容。 } \\
\hline 类别 & 内容 & 结构及内容 \\
\hline 数据记录日志 & $\begin{array}{l}\text { APP开启/关闭 } \\
\text { Service 查找与连接 } \\
\text { Characteristic查找结果 } \\
\text { Descriptor 写入记录 } \\
\text { 发送命令 } \\
\text { 下位机命令确认 } \\
\text { 下位机阻力参数上传 } \\
\text { APP开启/关闭 } \\
\text { 数据记录 }\end{array}$ & $\begin{array}{l}\text { app start/stop } \\
\text { service connected } \\
\text { 或 } \\
\text { service not found } \\
\text { characteristic connected + UUID } \\
\text { 或 } \\
\text { UUID failed } \\
\text { descriptor write } 0100 \text { succeed } \\
\text { ORDER: +命令内容 } \\
\text { Received: + 命令型 } \\
\text { received set: + 1档-2档。o。 } \\
\text { app start/stop } \\
\text { ResC + Res + Fre + AUTO }\end{array}$ \\
\hline
\end{tabular}

\subsection{2. 蓝牙设置模块}

蓝牙设置模块主要负责BLE从机设备的搜索和设备 信息的传递。界面如图4所示。

在点击“扫描”按键后, 设备开始搜索周围可用的BLE 从机设备, 并将其MAC地址和设备名称显示在窗口中共 选择[10]。通过点击列表中的名称来完成设备的选择, 按 下“选择”按键后, 将设备信息传递给主窗口以进行后续的 操作。

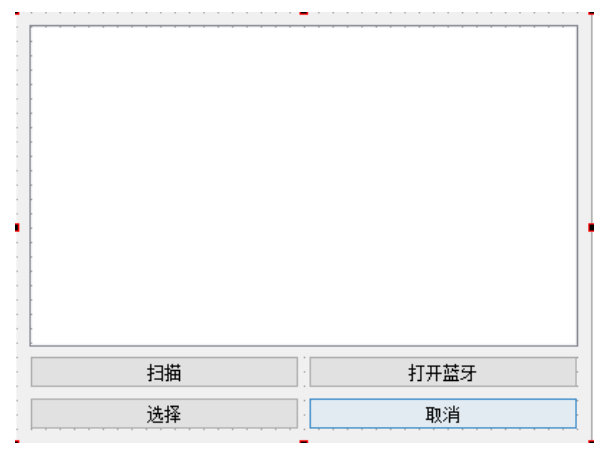

图4 蓝牙设置模块窗口图。 


\subsection{3．总控窗口}

总控模块包含目前所设计的所有控制命令, 通过主窗 口做中间介质与下位机通讯。其窗口如图5所示, 显示内 容包括当前阻力参数、步频档位和阻力参数组。按键包括 六项控制命令、阻力设置窗口打开和返回。

其具体内容见表4。

总控模块的显示内容是从下位机上传经主窗口处理 后以共有成员函数的形式传递至总控模块; 控制命令没有 实际内容, 只通过信号-槽机制触发主窗口的接收槽和命 令发送函数, 然后通过BLE Service传递给下位机。

表4 主窗口与总控窗口的信息交互内容。

\begin{tabular}{lll}
\hline 传递方向 & 内容 & 形式 \\
\hline \multirow{4}{*}{ 发送 } & 实时参数 & WD_controlcenter->setData(res, \\
& fre); & WD_controlcenter->getNRFRes(r \\
& 当前阻力参数设置 & esList); \\
接收 & 控制命令 & 信号-槽机制 \\
& 阻力设置 & 信号-槽机制 (含参) \\
\hline
\end{tabular}

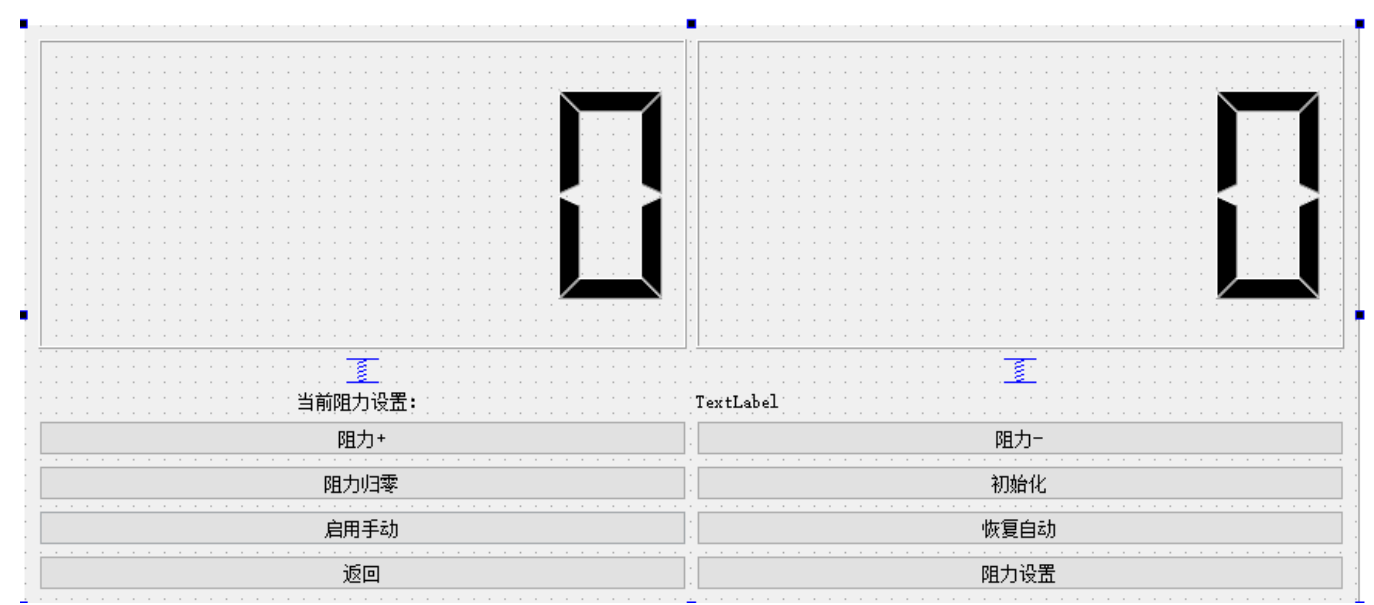

图5 控制窗口。

总控模块与阻力设置模块的信息交互见表 5 。

表5 总控模块与阻力设置模块的信息交互。

\begin{tabular}{llll}
\hline 传递方向 & 信息内容 & 格式 & 长度 \\
\hline 发送 & 当前阻力 & QList $<$ int $>$ & 10 \\
接收 & 设置阻力 & QList $<$ int $>$ & 10 \\
\hline
\end{tabular}

\subsection{4. 阻力设置模块}

阻力设置模块负责调节下位机的阻力参数组设置, 窗 口如图6所示。界面包含十个滑动条和三个功能按键。确
认按键按下后将发送当前十个滑动条的值至主窗口, 默认 按键按下后将调整十个滑动条至默认值, 取消按键按下后 直接返回上一层。

窗口中每个滑动条对应一档阻力参数, 通过拖动滑动 条来改变对应档位的阻力参数, 滑动范围为 $30-70$, 当释 放滑动条时, 若最终值小于上一档的值, 则会自动调整至 和上一档相等, 发送最终设置时, 会进行第二次轮询比较 确保阻力参数的设置不会随档位上升而下降。

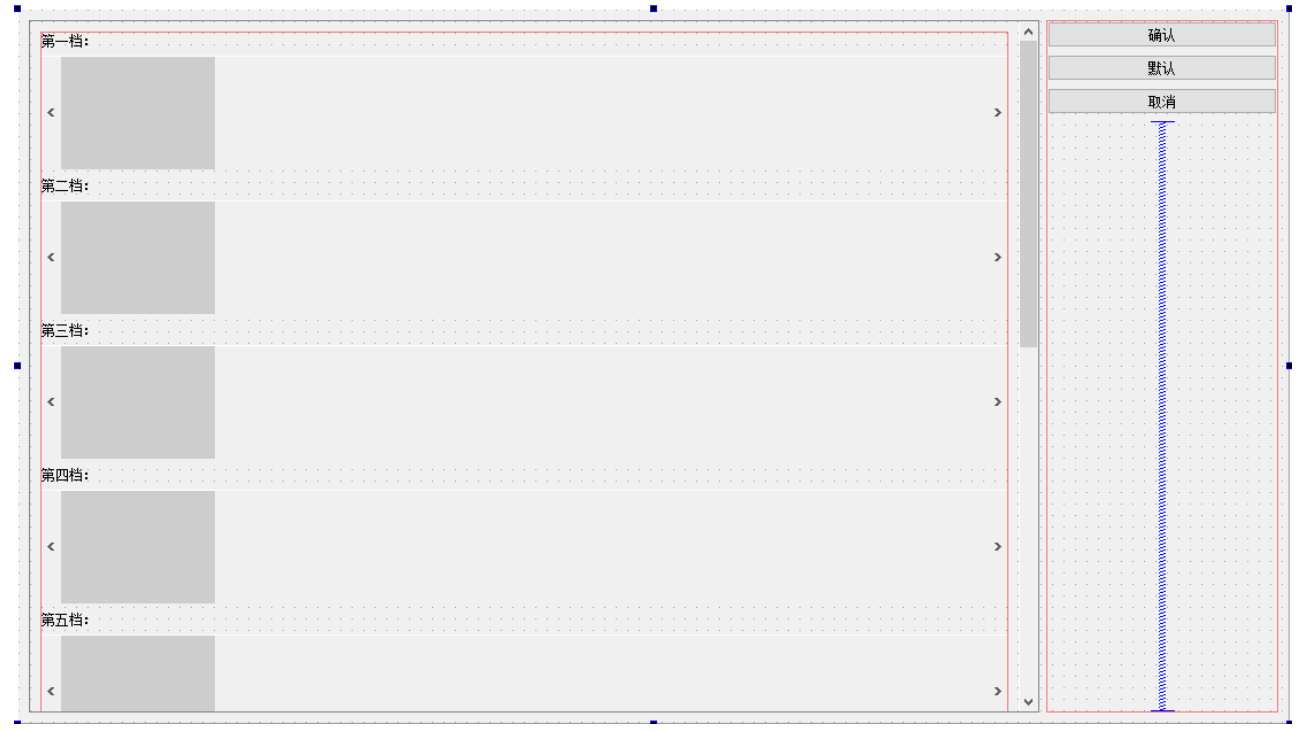

图6 阻力设置窗口。 


\section{4. 嵌入式硬件开发与软件设计}

本文嵌入式硬件电路以nRF51822(C)为核心，外围电 路包括电源模块、 $\mathrm{H}$ 桥电机驱动模块和输入输出模块, 机
械部分非本文研究内容, 不做阐述。假肢部分结构如图7 所示。

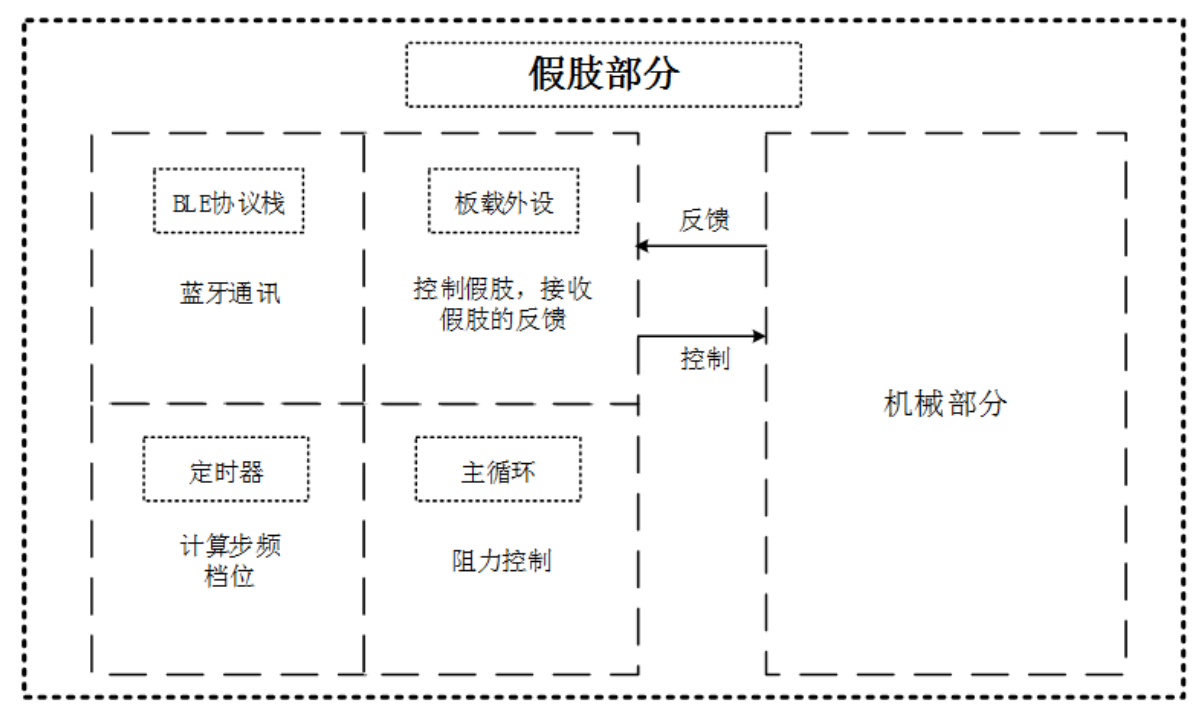

图7 下位机的架构系统工作流程。

\subsection{1. 初始化}

系统在上电之后经一系列初始化操作再进入主循环, 其初始化流程如图8所示。

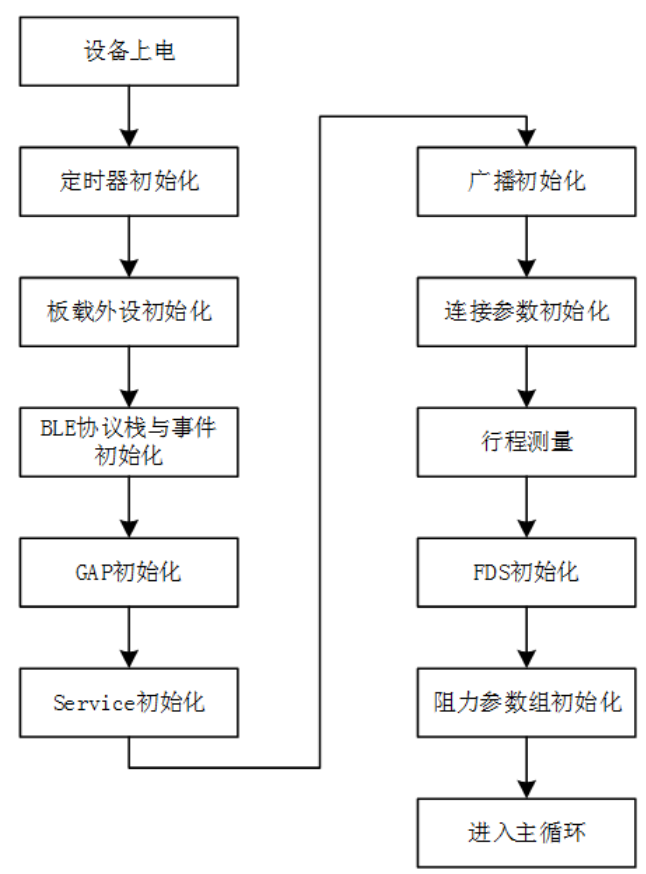

图8 初始化流程图。

定时器初始化包括系统APP Timer模块初始化和程序 中使用的APP Timer实例初始化, 此处用来设定Timer的ID、 模式和回调函数。板载外设初始化用来初始化默认的LED 和按键接口, 负责与假肢机械部分的控制信号的发送以及 反馈信号的接收。BLE协议栈与事件初始化负责BLE蓝牙
协议栈设置的初始化以及系统和BLE事件的分派函数设 置[11]。GAP初始化负责通用访问配置文件的初始化, 包 括设备名称、最小连接间隔、最大连接间隔、超时时间等。 Service初始化负责设置串口服务及其数据处理回调函数。 广播初始化负责初始化广播参数设置, 包括广播模式、设 备名称、UUID、广播时间、广播间隔等 $[12]$ 。连接参数初 始化负责设备与上位机进行BLE蓝牙连接时的参数设置。 行程测量用于测量电机旋转一周所用时间, 后续阻力调整 均基于此时间来计算调整时间。FDS初始化负责FDS模块 的设置, 主要用于储存阻力参数组等需要读写Flash的操作 的前置操作。阻力参数组初始化负责读取Flash中储存的阻 力参数组设置, 如果没有则取默认值。

\subsection{2. 主循环}

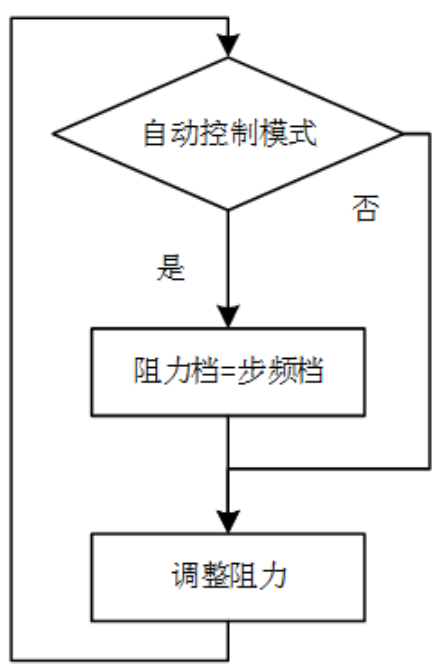

图9 主循环流程。 
系统主循环负责监控自动控制标志位的变化, 当自动 控制标志位置1时, 表示进入自动控制模式, 此时的阻力 档位将根据预设和实时步频进行自动调整; 当自动控制标 志位置 0 时, 表示进入手动控制模式, 此时只能通过上位 机控制阻力档位。流程图如图9所示。

\subsection{3. 中断}

系统中断包含引脚中断、定时器中断、BLE Service 中断和FDS中断。其中, 引脚中断负责接收机械部分的反 馈信息; 定时器中断负责步频的相关计算和数据上传;

BLE Service中断负责处理BLE事件; FDS中断负责处理 Flash读写事件。

引脚中断处理流程如图10所示。接收到干簧管触发的 反馈信号时, 实时步数会增加 1 步, 用于定时器中断中的 步频计算; 接收到电机触碰开关反馈时, 表示电机已抵达 起点/终点, 此时根据算法来控制电机反转或停止。

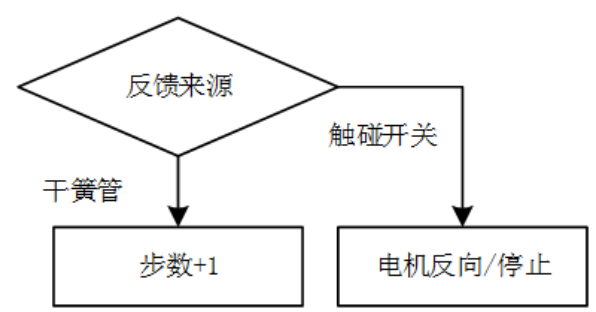

图10引脚中断处理逻辑。

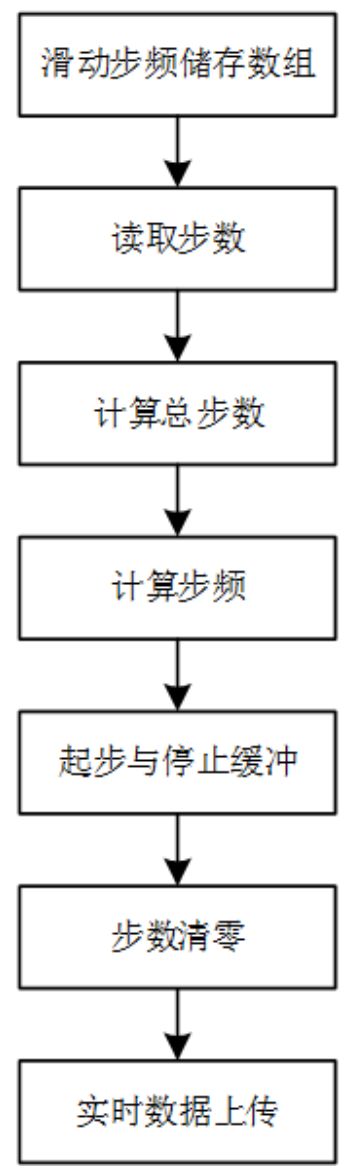

图11 定时器中断处理流程。
定时器中断处理流程如图11所示。计时器通过一个不 断滑动的数组来储存所有统计的步数。进入定时器中断回 调函数后, 首先将储存步数的数组依次前移一位, 并在最 后一位储存此时的步数, 并求出总和。然后根据该数组第 一位是否为 0 来判断时候进行步频计算, 根据总和是否为 0 来判断是否进行起步、静止缓冲。最后清零当前统计的步 数, 并通过BLE协议栈发送实时运动数据给上位机。

步频的计算流程如图12所示。当步数储存数组第一位 出现有效数字 (不为 0 ) 后, 开始查找下一个有效位 $\mathrm{i}$, 如 有，则两位之间的时间差即为两步之间的时间差 (精度为 定时器触发时间)。此外, 还要防止抖动带来的误差, 所 以当出现了较短的时间差 (<3档) 且步频档位跳动较大 (上 一次步频档 $<7$ ）时, 将此次判断为抖动, 并忽略。

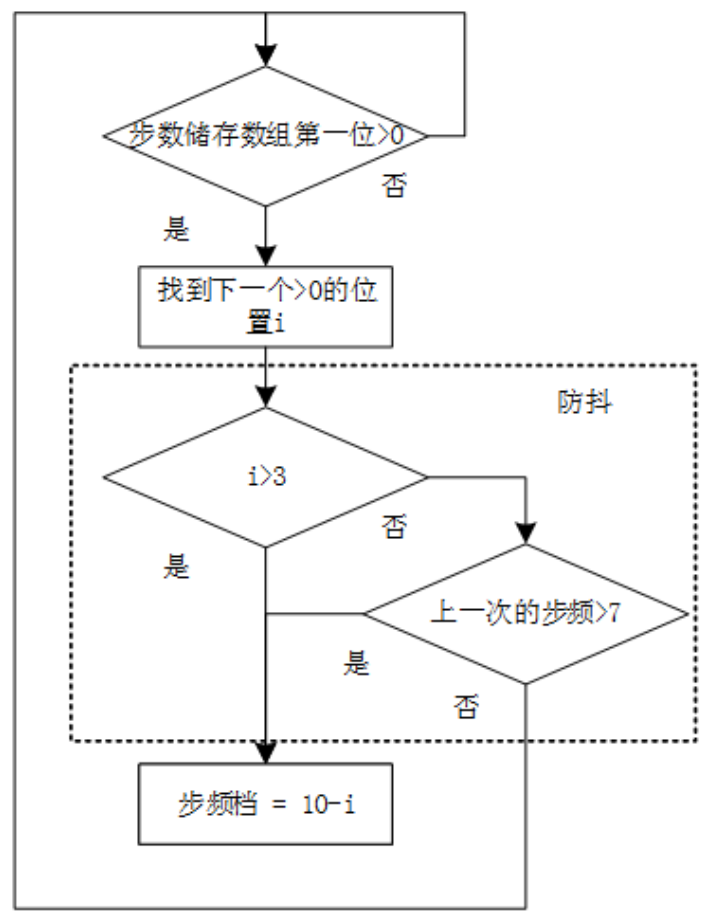

图12 步频计算流程。

起步及停止时的缓冲处理流程如图13所示。

当总步数为 0 时, 考虑两种状态: 长时间静止和刚刚 进入静止的短时静止状态。这里通过累计静止的时间来区 分这两种状态。当总数为 0 且累计静止时间未超出阈值时, 认为处于短时静止状态, 此时将步频档设为 2 , 通过主循 环设置阻力档和步频档相等来达到短时静止时仍有 2 档阻 力的目的。档总数为 0 且累计静止时间超出阈值时, 认为 处于长时间静止状态, 此时步频置为 0 。

当总数不为 0 时, 考虑两种状态: 正常运动和刚刚进 去运动的第一步到第二步之间。由于步频计算算法的限制, 只有在接收到第二步的反馈后才能计算出步频, 然后进行 阻力的调整, 起步阶段则无法及时给一个缓冲的档位, 所 以需要对以上两种状态进行区分。这里通过储存上一次的 运动状态来进行起步缓冲。当总数不为 0 且上一次的运动 状态为静止时, 认为此时产生了第一步运动, 则将步频调 整为 2 档, 继而通过主循环调整阻力为 2 档, 达到起步缓冲 的目的。 


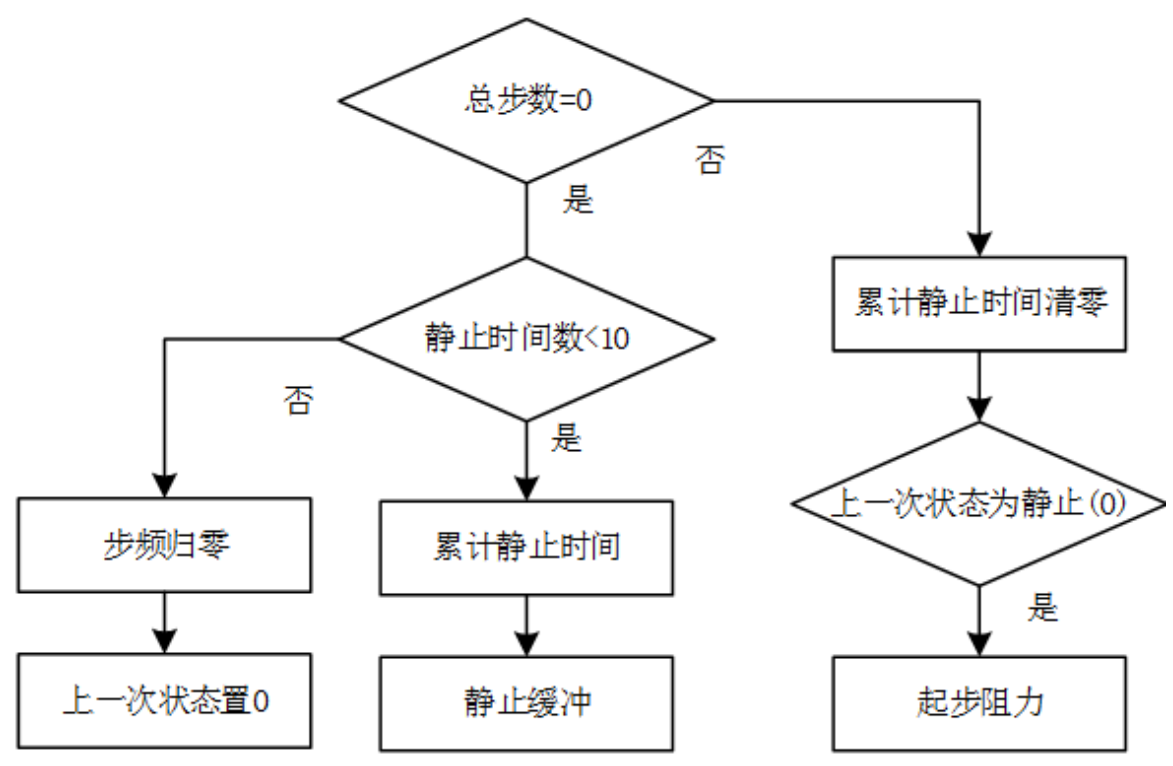

图13 起步与停止缓冲处理流程。

FDS中断负责处理上位机传来的阻力参数组设置, 对 储存在本地Flash上的数据进行更新。

\subsubsection{FDS}

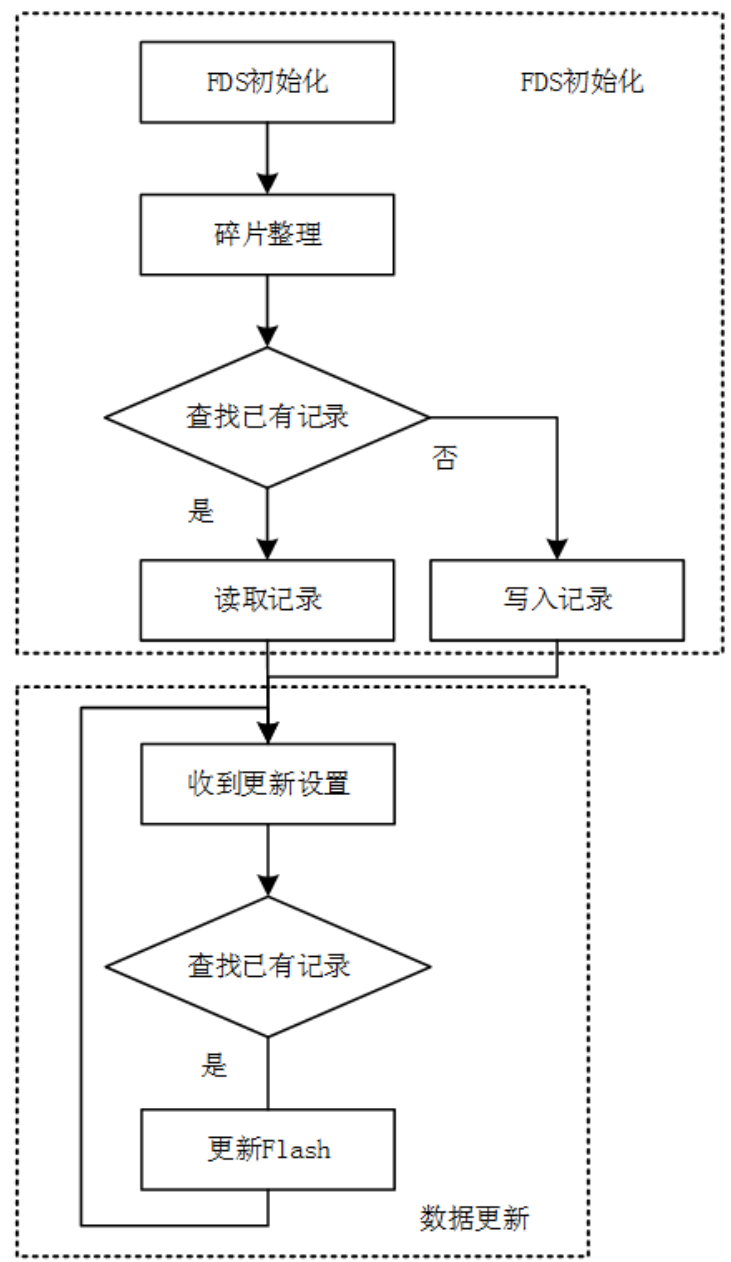

图14 FDS的工作流程。
为了便于用户根据各自的情况修改阻力参数, 通过 FDS模块来处理上位机发来的阻力参数设置。FDS的工作 流程如图14所示。FDS初始化包括: FDS模块初始化、碎 片收集、查找记录和读取或写入记录, 在进入主循环之前 的系统初始化过程中完成。FDS模块初始化负责初始化 Flash读写功能; 碎片收集负责清理空间, FDS模块的删除 功能并不会删除数据的物理内容, 只是修改了其标记, 仍 会占用空间, 所以需要定时清理删除内容所占用的空间; 查找记录负责查找Flash中是否已经写入数据, 有则读取, 无则写入默认参数 [13]。数据更新包括查找记录和更新数 据, 在FDS中断中完成。在更新指定Flash之前需要获取该 段数据的key, 然后再进行更新, 同样, 此处不会删除旧 数据, 只能通过碎片收集来清理空间。

\section{2. 阻力调整}

系统调整阻力的流程如图15所示。

每次当主循环进入阻力调整函数后, 依次判断阻力是 否不变、是否调整至 0 , 然后进行相应的调整。由于对电 机的控制是一个开环控制, 不具有反馈信息, 无法知道电 机具体转到的位置, 只能在收到电机触发触碰开关后知道 电机已抵达零点 $[14]$ 。所以在连续调整阻力的过程中, 不 可避免的遇到阻力调整不够精确且误差逐渐累计的问题, 必须对误差进行处理。这里, 利用电机旋转至零点时触发 的触碰开关来保证阻力归零时电机位置也归零, 即可在阻 力归零时清理积累的误差。

电机控制参数计算流程如图16所示。通过计算当前和 目标档位所对应的时间参数之差, 乘以电机转动一周时间 的 $1 / 2$, 即为最终转动时间。

电机控制流程如图17所示。计算得到电机转动方向和 转动时间后, 通过延时和控制引脚高低来达到最终的控制 效果。 


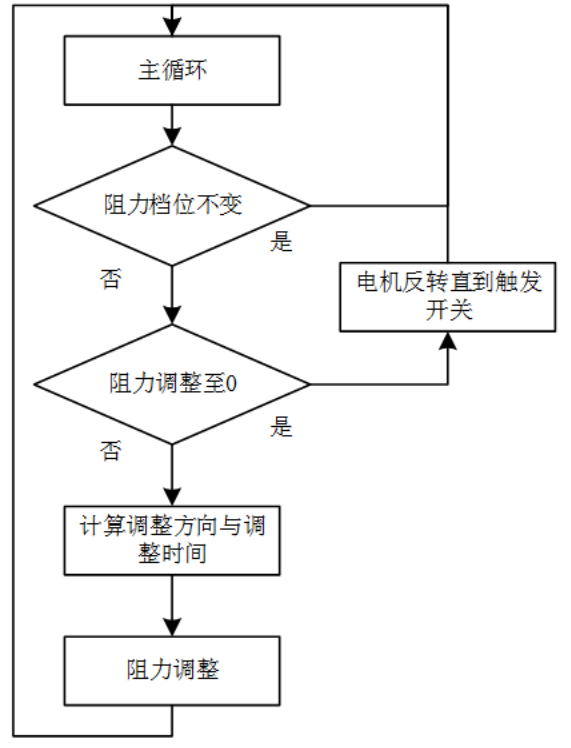

图15 阻力调整流程。

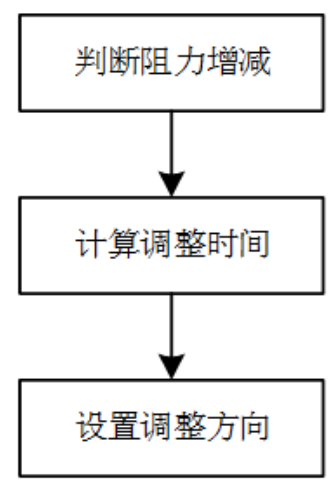

图16 电机控制参数计算流程。

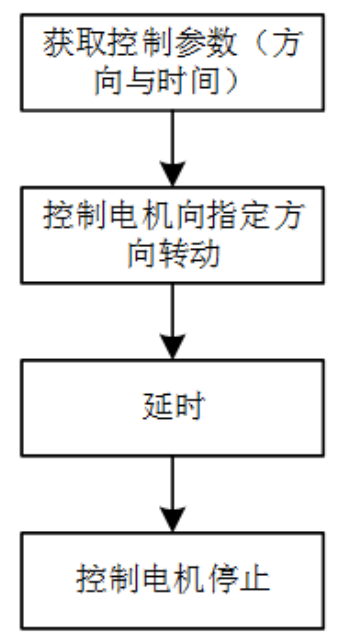

图17 电机控制流程。

\section{3. 电路部分}

嵌入式电路部分的结构图如图18所示, 包括了程序烧 录电路、nRF51822最小系统电路、电源稳压电路、反馈电 路、电机控制电路。

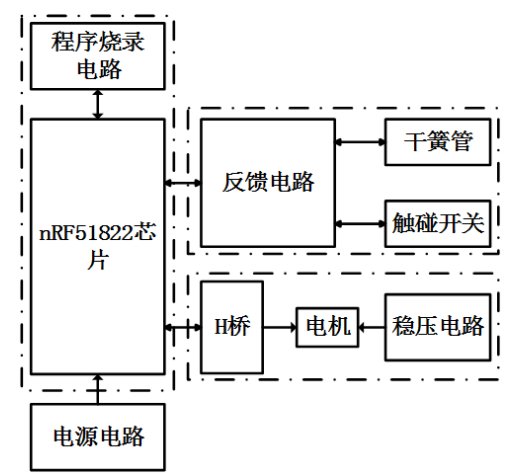

图18 电路结构图。

\section{5. 实验结果与分析}

\section{1. 设备连接与控制测试}

蓝牙扫描结果如图19所示, 可以扫描到设备。蓝牙连 接后可实时显示各种状态信息, 如图20所示。Android APP 日志记录结果如图21所示。

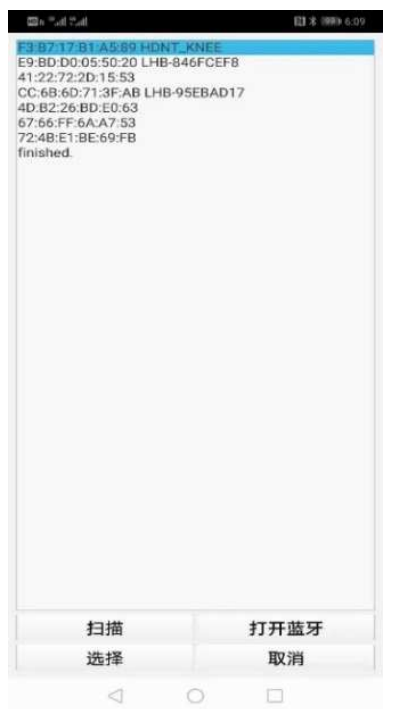

图19 蓝牙扫描结果。

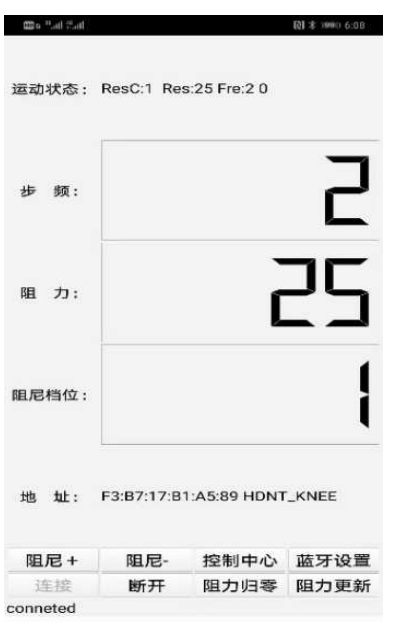

图20 主窗口实时显示步频、阻力参数、设备信息、连接状态。 

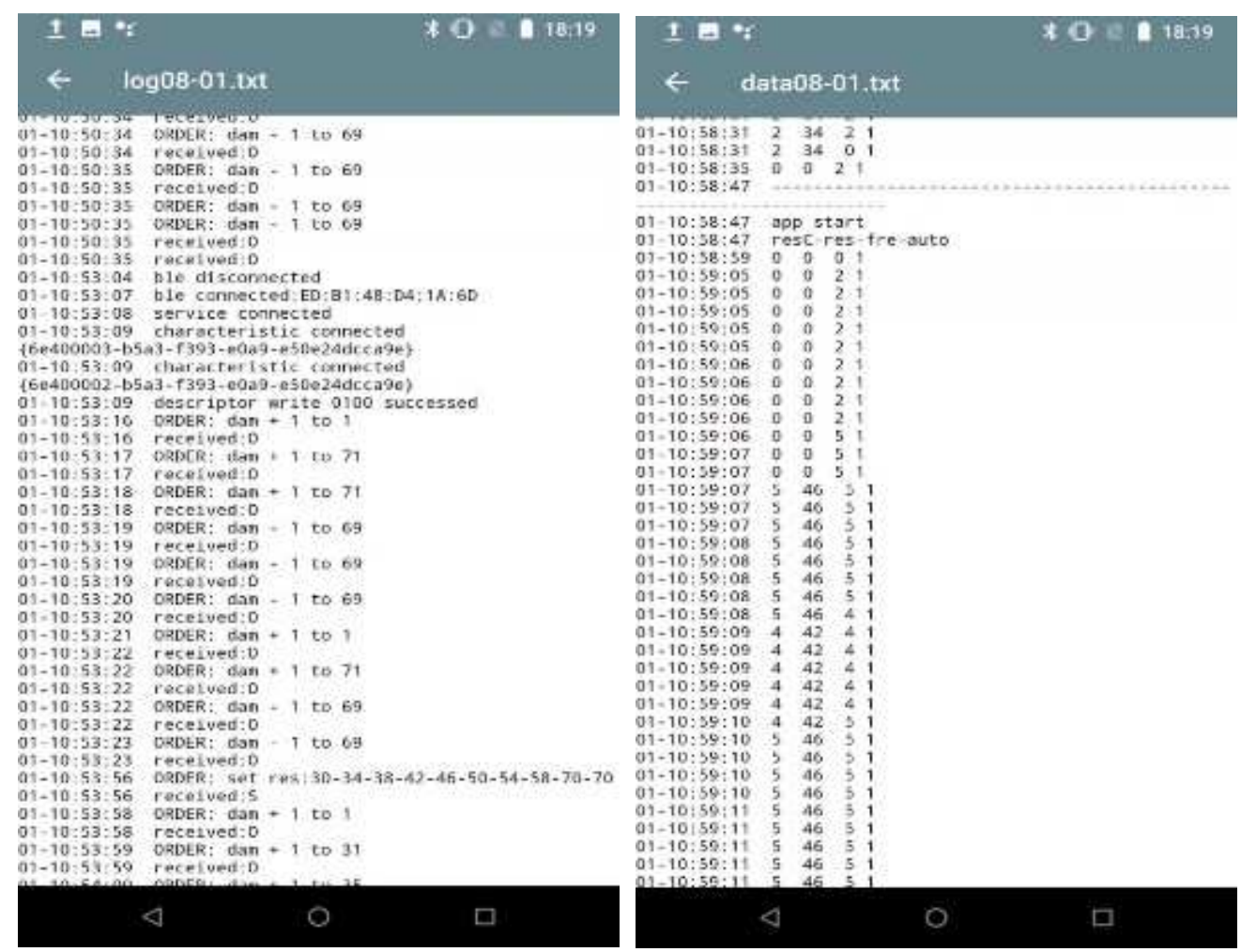

图21 系统控制日志与数据日志。

\section{2. 助力控制测试}

图22是设备助力控制测试实验中的步频档位-助力档 位曲线。分为两部分, 第一部分步频档和对应的助力档对 等, 第二部分将助力档整体提升了一档。可以看出, 助力
可以根据步频的变化进行实时的调整, 且具体步频对应的 档位可以根据每个用户不同的行走习惯在Android端远程 修改。

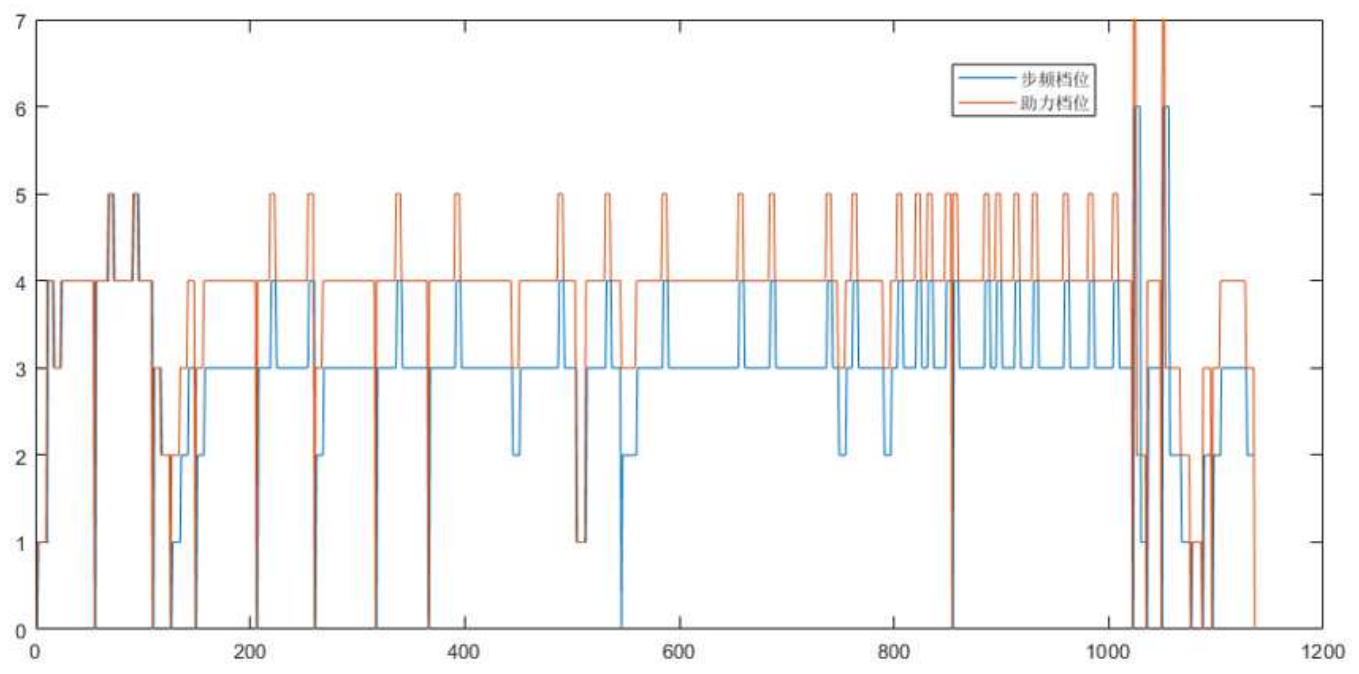

图22 步频档位-助力档位曲线。

\section{6. 结论}

针对传统电动助力膝关节成本高、非智能、无法自动 调节助力强度的问题, 本文研制了一种新的电动助力膝关 节控制系统，系统分为Android控制终端、假肢控制模块、
假肢机械结构三部分。Android控制终端通过蓝牙与假肢 控制模块进行数据交互, 控制机械部分运动, 进行助力行 走。经过实验测试, 设备可以正常运行, 且使用者可以根 据身体运动情况手动控制助力大小, 调整至最佳运行状态。 且系统也能够根据实时步速自动调节膝关节助力大小。 


\section{参考文献}

[1] 中华人民共和国国家统计局, 第二次全国残疾人调查领导 小组. 《2006年第二次全国残疾人抽样调查主要数据公报》 ( 第 一 号 )[EB/OL]. (2007-11-21). [2015-02-23]. http://www.cdpf.org.cn/sjzx/cjrgk/200804/t20080407_387580 .shtml.

[2] 杨鹏, 刘作军, 耿艳利, 等. 智能下肢假肢关键技术研究进 展 $[\mathrm{J}]$. 河北工业大学学报, 2013(1):76-80.

[3] 王文俊. 基于映射模型的假肢膝关节控制方法研究 [D]. 苏 州: 苏州大学, 2013.

[4] 马玉良, 徐文良, 孟明, 等. 基于神经网络的智能下肢假肢 自适应控制 [J]. 浙江大学学报：工学版，2010, 44(7):1373-1376.

[5] 喻洪流. 假肢矫形器原理与应用 $[\mathrm{M}]$. 南京: 东南大学出版 社, 2011, 104-105.

[6] 张宁, 李剑. 动力型智能假肢膝关节的研究进展 [J]. 北京 生物医学工程, 2018, v.37(04):101-106.

[7] Ensworth J F, Reynolds M S. BLE-Backscatter: Ultralow-Power IoT Nodes Compatible With Bluetooth 4.0 Low Energy (BLE) Smartphones and Tablets[J]. IEEE

Transactions on Microwave Theory \& Techniques, 2017, PP(99):1-9.

[8] Ensworth J F, Hoang A T, Phu T Q, et al. Full-duplex Bluetooth Low Energy (BLE) compatible Backscatter communication system for mobile devices[C]. Wireless Sensors \& Sensor Networks. 2017.

[9] Mackensen E, Lai M, Wendt T M. Bluetooth Low Energy (BLE) based wireless sensors[C]. Sensors. 2012.

[10] Gomez C, Oller J, Paradells J. Overview and Evaluation of Bluetooth Low Energy: An Emerging Low-Power Wireless Technology[J]. Sensors, 2012, 12(9):11734-11753.

[11] 范晨灿. 基于蓝牙 4.0传输的Android手机心电监护系统[D]. 浙江大学.

[12] Dai Y, Jian L. Design of Noninvasive Pulse Oximeter Based on Bluetooth 4.0 BLE[C]. Seventh International Symposium on Computational Intelligence \& Design. 2015.

[13] 唐否. 基于FPGA的USB、Flash控制器设计 [D]. 北京交通大 学, 2010.

[14] 张梁, 朱明富. 一种感应式自适应触摸开关的设计 [J]. 自 动化与仪表, 2007, 22(5):27-29. 